\title{
A GENERAL THEOREM CONCERNING THE STABILITY OF A PARTICULAR NON-NEWTONIAN FLUID*
}

\author{
BY \\ SAMUEL M. GENENSKY \\ Mathematics Division, The RAND Corporation
}

Summary. It is the intention of the present paper to prove a theorem concerning the stability of a particular non-Newtonian fluid suggested to the author by Professor R. S. Rivlin of Brown University. The method used in proving this theorem is similar to that employed by $\mathrm{H}$. Schlichting in his proof of a similar theorem for an inviscid fluid which was originally established by Lord Rayleigh. The acceleration gradients introduced by the non-Newtonian fluid model into the constitutive equations are found to alter the stability criterion set forth by Rayleigh for an inviscid fluid.

1. Introduction. As early as 1880 Rayleigh [1] proved that for an inviscid fluid the existence of a point of inflection in the velocity profile of a steady one-dimensional basic flow is a necessary condition for the growth of a superimposed two-dimensional disturbance. It is the intention of the present paper to prove a similar theorem for a particular non-Newtonian fluid suggested to the author by Professor R. S. Rivlin.* The method used in proving this theorem is similar to that employed by Schlichting [2] in his proof of the Rayleigh theorem.

2. The constitutive equations and equations of motion. Let $X_{i}(j=1,2,3)$ be the coordinates referred to a rectangular Cartesian coordinate system $x_{i}$, of a generic particle of a continuous medium in the undeformed state at time $t_{0}$. Let $x_{i}$ be the coordinates of the same particle in the deformed state at time $t$. It then follows that the components of velocity $v_{i}$ and the components of acceleration $a_{i}$ of the particle are given by

$$
v_{i}=\frac{\partial x_{i}}{\partial t} \quad \text { and } \quad a_{i}=\frac{\partial^{2} x_{i}}{\partial t^{2}},
$$

where $x_{i}$ are regarded as single-value continuous functions of $X_{k}(k=1,2,3)$ and $t$, having as many continuous derivatives as the analysis requires. As is well known, if $v_{i}$ are considered to be functions of $x_{k}$ and $t$, then

$$
a_{i}=\frac{\partial v_{i}}{\partial t}+v_{k} \frac{\partial v_{j}}{\partial x_{k}}
$$

Now the equations of motion are

$$
\rho\left(\frac{\partial v_{i}}{\partial t}+v_{k} \frac{\partial v_{i}}{\partial x_{k}}\right)+\rho f_{i}=\frac{\partial t_{i l}}{\partial x_{l}}, \quad(l=1,2,3),
$$

where $\rho$ is the mass of the medium per unit volume and $f_{i}$ are the components in the coordinate directions of the body force per unit mass, also measured in the deformed state. The components of stress $t_{i l}$ resulting from the deformation are defined as follows; $t_{i 1}, t_{i 2}$ and $t_{i 3}$ are the components of the force per unit area in the positive direction of the $x_{1}, x_{2}$ and $x_{3}$ axes respectively, measured in the deformed state, exerted across an

${ }^{1}$ Received October 28, 1958; revised manuscript received August 12, 1959. 
element of area at $\left(x_{1}, x_{2}, x_{3}\right)$ normal to the $x_{i}$ axis, by the material on the positive side of the element upon the material on the negative side of the element.

Rivlin [3] showed that if $t_{i l}$ at the point $x_{k}$ and at time $t$ are assumed to be polynomials in the velocity gradients $\partial v_{m} / \partial x_{n}(m, n=1,2,3)$ and the acceleration gradients $\partial a_{m} / \partial x_{n}$ and if, in addition, the medium is assumed to be isotropic at time $t_{0}$, then the stress matrix $\mathbf{T}=\left\|t_{i l}\right\|$ is expressible in the form

$$
\begin{aligned}
\mathbf{T}=\varphi_{0} \mathbf{I}+\varphi_{1} \mathbf{A}_{1} & +\varphi_{2} \mathbf{A}_{2}+\varphi_{3} \mathbf{A}_{1}^{2}+\varphi_{4} \mathbf{A}_{2}^{2}+\varphi_{5}\left(\mathbf{A}_{1} \mathbf{A}_{2}+\mathbf{A}_{2} \mathbf{A}_{1}\right) \\
& +\varphi_{6}\left(\mathbf{A}_{1}^{2} \mathbf{A}_{2}+\mathbf{A}_{2} \mathbf{A}_{1}^{2}\right)+\varphi_{7}\left(\mathbf{A}_{1} \mathbf{A}_{2}^{2}+\mathbf{A}_{2}^{2} \mathbf{A}_{1}\right)+\varphi_{8}\left(\mathbf{A}_{1}^{2} \mathbf{A}_{2}^{2}+\mathbf{A}_{2}^{2} \mathbf{A}_{1}^{2}\right),
\end{aligned}
$$

where $I$ is the unit matrix, $\mathbf{A}_{1}$ and $\mathbf{A}_{2}$ are symmetric kinematic matrices defined by

$$
\mathbf{A}_{1}=\left\|\frac{\partial v_{i}}{\partial x_{l}}+\frac{\partial v_{l}}{\partial x_{i}}\right\| \text { and } \mathbf{A}_{2}=\left\|\frac{\partial a_{i}}{\partial x_{l}}+\frac{\partial a_{l}}{\partial x_{i}}+2 \frac{\partial v_{m}}{\partial x_{j}} \frac{\partial v_{m}}{\partial x_{l}}\right\|
$$

and $\varphi_{\mathbf{q}}(q=0,1,2, \cdots, 8)$ are polynomials in $\operatorname{tr} \mathbf{A}_{1}, \operatorname{tr} \mathbf{A}_{2}, \operatorname{tr} \mathbf{A}_{1}^{2}, \operatorname{tr} \mathbf{A}_{2}^{2}, \operatorname{tr} \mathbf{A}_{1}^{3}, \operatorname{tr} \mathbf{A}_{2}^{3}, \operatorname{tr} \mathbf{A}_{1} \mathbf{A}_{2}$, $\operatorname{tr} \mathbf{A}_{1}^{2} \mathbf{A}_{2}, \operatorname{tr} \mathbf{A}_{1} \mathbf{A}_{2}^{2}$ and $\operatorname{tr} \mathbf{A}_{1}^{2} \mathbf{A}_{2}^{2}$. In a later paper Rivlin [4] pointed out that for an incompressible material, the stress corresponding to a specific state of flow is indeterminate to the extent of an arbitrary hydrostatic pressure $p$. Since in the present paper we shall confine our analysis to an incompressible fluid, we may replace $\varphi_{0}$ in equation (2.4) by - $p$. We shall also restrict our investigation to a fluid for which $\varphi_{1}$ and $\varphi_{2}$ are constants and $\varphi_{a}(q=3,4,5, \cdots, 8)$ are identically zero.

Equation (2.4) now takes the form

$$
\mathbf{T}=-p \mathbf{I}+\varphi_{1} \mathbf{A}_{1}+\varphi_{2} \mathbf{A}_{2}
$$

or alternatively

$$
t_{i l}=-p \delta_{i l}+\varphi_{1}\left(\frac{\partial v_{i}}{\partial x_{l}}+\frac{\partial v_{l}}{\partial x_{i}}\right)+\varphi_{2}\left(\frac{\partial a_{i}}{\partial x_{l}}+\frac{\partial a_{l}}{\partial x_{i}}+2 \frac{\partial v_{m}}{\partial x_{i}} \frac{\partial v_{m}}{\partial x_{l}}\right) .
$$

Introducing Eqs. (2.2) into Eqs. (2.7), we obtain

$$
\begin{aligned}
t_{i l}=-p \delta_{i l}+ & \varphi_{1}\left(\frac{\partial v_{i}}{\partial x_{l}}+\frac{\partial v_{l}}{\partial x_{i}}\right)+\varphi_{2}\left(\frac{\partial^{2} v_{j}}{\partial t \partial x_{l}}+\frac{\partial v_{m}}{\partial x_{l}} \frac{\partial v_{j}}{\partial x_{m}}\right. \\
& \left.+v_{m} \frac{\partial^{2} v_{j}}{\partial x_{l} \partial x_{m}}+\frac{\partial^{2} v_{l}}{\partial t \partial x_{i}}+\frac{\partial v_{m}}{\partial x_{i}} \frac{\partial v_{l}}{\partial x_{m}}+v_{m} \frac{\partial^{2} v_{l}}{\partial x_{i} \partial x_{m}}+2 \frac{\partial v_{m}}{\partial x_{i}} \frac{\partial v_{m}}{\partial x_{l}}\right) .
\end{aligned}
$$

Since we have assumed that the fluid is incompressible, the continuity equation is

$$
\frac{\partial v_{m}}{\partial x_{m}}=0 \text {. }
$$

Introducing the constitutive equations (2.8) in the equations of motion (2.3), employing the incompressibility condition (2.9) and neglecting body forces, we obtain

$$
\begin{aligned}
\rho\left(\frac{\partial v_{i}}{\partial t}+v_{k} \frac{\partial v_{i}}{\partial x_{k}}\right)=-\frac{\partial p}{\partial x_{i}}+\varphi_{1} \frac{\partial^{2} v_{i}}{\partial x_{l} \partial x_{l}}+\varphi_{2} & \frac{\partial^{3} v_{i}}{\partial t \partial x_{l} \partial x_{l}} \\
& +\frac{\partial v_{i}}{\partial x_{m}} \frac{\partial^{2} v_{m}}{\partial x_{l} \partial x_{l}}+2 \frac{\partial v_{m}}{\partial x_{l}} \frac{\partial^{2} v_{i}}{\partial x_{l} \partial x_{m}} \\
& +v_{m} \frac{\partial^{3} v_{j}}{\partial x_{l} \partial x_{l} \partial x_{m}}+2 \frac{\partial v_{m}}{\partial x_{l}} \frac{\partial^{2} v_{l}}{\partial x_{i} \partial x_{m}} \\
& \left.+2 \frac{\partial v_{m}}{\partial x_{l}} \frac{\partial^{2} v_{m}}{\partial x_{i} \partial x_{l}}+2 \frac{\partial v_{m}}{\partial x_{i}} \frac{\partial^{2} v_{m}}{\partial x_{l} \partial x_{l}}\right) .
\end{aligned}
$$


3. Development of the stability equation. Consider next a two-dimensional steady laminar flow with velocity components

$$
W_{1}=W_{1}\left(x_{2}\right) \text { and } W_{2}=W_{3}=0 .
$$

Examples of such flows are the flow between a pair of parallel plates sufficiently removed from the intake section, and the flow in the boundary layer along a flat plate excluding the region of its leading edge. The pressure $P$ necessary to maintain such flows is in general a function of both $x_{1}$ and $x_{2}$.

We shall superimpose upon the laminar flow a small two dimensional disturbance with velocity components and associated pressure given by

$$
u_{1}=u_{1}\left(x_{1}, x_{2}, t\right), \quad u_{2}=u_{2}\left(x_{1}, x_{2}, t\right), \quad u_{3}=0 \text { and } p^{*}=p^{*}\left(x_{1}, x_{2}, t\right) \text {. }
$$

Thus the velocity components and pressure of the composite flow are

$$
v_{1}=W_{1}+u_{1}, \quad v_{2}=u_{2}, \quad v_{3}=0 \text { and } p=P+p^{*} .
$$

Further, we shall require that the composite flow satisfy the same boundary conditions as the steady laminar flow. Thus the disturbance satisfies the boundary conditions

$$
u_{1}=u_{2}=0 \text { at } x_{2}=0 \text { and } x_{2}=L
$$

in the case of flow between parallel plates separated by a distance $L$, and

$$
u_{1}=u_{2}=0 \text { at } x_{2}=0 \text { and } x_{2} \rightarrow \infty
$$

in the case of boundary layer flow along a flat plate.

Next, introducing the velocity components and pressure given by (3.3) into Eqs. (2.9) and (2.10), and assuming that the velocity components $u_{i}$ are sufficiently small so that terms of the second degree in $u_{i}$ and derivatives of $u_{i}$ may be neglected in comparison with terms of the first degree, we obtain

$$
\begin{aligned}
& \frac{\partial u_{1}}{\partial x_{1}}+\frac{\partial u_{2}}{\partial x_{2}}=0, \\
& \rho\left(\frac{\partial u_{1}}{\partial t}+W_{1} \frac{\partial u_{1}}{\partial x_{1}}+W_{1}^{\prime} u_{2}\right)=-\frac{\partial P}{\partial x_{1}}-\frac{\partial p^{*}}{\partial x_{1}}+\varphi_{1}\left(\nabla^{2} u_{1}+W_{1}^{\prime \prime}\right) \\
& +\varphi_{2}\left(\nabla^{2} \frac{\partial u_{1}}{\partial t}+2 W_{1}^{\prime \prime} \frac{\partial u_{2}}{\partial x_{2}}+4 W_{1}^{\prime} \frac{\partial^{2} u_{1}}{\partial x_{1} \partial x_{2}}\right. \\
& +W_{1}^{\prime} \nabla^{2} u_{2}+3 W_{1}^{\prime \prime} \frac{\partial u_{1}}{\partial x_{1}}+W_{1} \nabla^{2} \frac{\partial u_{1}}{\partial x_{1}} \\
& \left.+W_{1}^{\prime \prime \prime} u_{2}+2 W_{1}^{\prime} \frac{\partial^{2} u_{2}}{\partial x_{1}^{2}}\right)
\end{aligned}
$$

and

$$
\begin{aligned}
\rho\left(\frac{\partial u_{2}}{\partial t}+W_{1} \frac{\partial u_{2}}{\partial x_{1}}\right)=-\frac{\partial P}{\partial x_{2}} & -\frac{\partial p^{*}}{\partial x_{2}}+\varphi_{1} \nabla^{2} u_{2}+\varphi_{2}\left(\nabla^{2} \frac{\partial u_{2}}{\partial t}\right. \\
& +4 W_{1}^{\prime} \frac{\partial^{2} u_{2}}{\partial x_{1} \partial x_{2}}+3 W_{1}^{\prime \prime} \frac{\partial u_{2}}{\partial x_{1}}+W_{1} \nabla^{2} \frac{\partial u_{2}}{\partial x_{1}} \\
& \left.+4 W_{1}^{\prime} W_{1}^{\prime \prime}+4 W_{1}^{\prime \prime} \frac{\partial u_{1}}{\partial x_{2}}+2 W_{1}^{\prime} \frac{\partial^{2} u_{1}}{\partial x_{2}^{2}}+2 W_{1}^{\prime} \nabla^{2} u_{1}\right),
\end{aligned}
$$


where $\nabla^{2}=\left(\partial^{2} / \partial x_{1}^{2}\right)+\left(\partial^{2} / \partial x_{2}^{2}\right)$, and primes denote ordinary differentiation with respect to $x_{2}$.

Further, introducing Eq. (3.6) into Eqs. (3.7) and (3.8) and assuming the basic laminar flow satisfies the equations of motion, we obtain

$$
\begin{aligned}
\rho\left(\frac{\partial u_{1}}{\partial t}+W_{1} \frac{\partial u_{1}}{\partial x_{1}}\right. & \left.+W_{1}^{\prime} u_{2}\right)=-\frac{\partial p^{*}}{\partial x_{1}}+\varphi_{1} \nabla^{2} u_{1}+\varphi_{2}\left(\nabla^{2} \frac{\partial u_{1}}{\partial t}\right. \\
& \left.+3 W_{1}^{\prime} \frac{\partial^{2} u_{1}}{\partial x_{1} \partial x_{2}}+W_{1}^{\prime \prime} \frac{\partial u_{1}}{\partial x_{1}}+W_{1} \nabla^{2} \frac{\partial u_{1}}{\partial x_{1}}+W_{1}^{\prime \prime \prime} u_{2}+3 W_{1}^{\prime} \frac{\partial^{2} u_{2}}{\partial x_{1}^{2}}\right)
\end{aligned}
$$

and

$$
\begin{aligned}
\rho\left(\frac{\partial u_{2}}{\partial t}+W_{1} \frac{\partial u_{2}}{\partial x_{1}}\right)=- & \frac{\partial p^{*}}{\partial x_{2}}+\varphi_{1} \nabla^{2} u_{2}+\varphi_{2}\left(\nabla^{2} \frac{\partial u_{2}}{\partial t}+2 W_{1}^{\prime} \frac{\partial^{2} u_{2}}{\partial x_{1} \partial x_{2}}\right. \\
& \left.+3 W_{1}^{\prime \prime} \frac{\partial u_{2}}{\partial x_{1}}+W_{1} \nabla^{2} \frac{\partial u_{2}}{\partial x_{1}}+4 W_{1}^{\prime \prime} \frac{\partial u_{1}}{\partial x_{2}}+4 W_{1}^{\prime} \frac{\partial^{2} u_{1}}{\partial x_{2}^{2}}\right) .
\end{aligned}
$$

Differentiating Eq. (3.9) with respect to $x_{2}$ and Eq. (3.10) with respect to $x_{1}$, eliminating $\partial^{2} p^{*} / \partial x_{1} \partial x_{2}$ from the resulting equations and again employing Eq. (3.6), we arrive at

$$
\left(\frac{\partial}{\partial t}+W_{1} \frac{\partial}{\partial x_{1}}-\nu \nabla^{2}-\gamma \nabla^{2} \frac{\partial}{\partial t}-\gamma W_{1} \nabla^{2} \frac{\partial}{\partial x_{1}}\right)\left(\frac{\partial u_{1}}{\partial x_{2}}-\frac{\partial u_{2}}{\partial x_{1}}\right)=\left(\gamma W^{\prime \prime \prime \prime}-W^{\prime \prime}\right) u_{2},
$$

where $\nu=\varphi_{1} / \rho$ and $\gamma=\varphi_{2} / \rho$.

Further, we shall assume that the velocity components of the disturbance are of the form

$$
u_{i}\left(x_{1}, x_{2}, t\right)=u_{i}^{*}\left(x_{2}\right) \exp \left[i A\left(x_{1}-C t\right)\right], \quad(j=1,2)
$$

where $\left|u_{i}^{*} u_{i}^{*}\right|$ is the amplitude and $A$ the wave number of the disturbance, and $C$ is a complex number. The real part of $C, C_{r}$, is the phase velocity of the disturbance and the imaginary part, $C_{i}$, is the amplification factor. If $C_{i}>0$, the disturbance tends to grow; if $C_{i}<0$, the disturbance decays; and if $C_{i}=0$, the disturbance is neutral.

Introducing the velocity components (3.12) into Eqs. (3.6) and (3.11), we obtain

$$
i A u_{1}^{*}+u_{2}^{* \prime}=0
$$

and

$$
\begin{aligned}
i A\left(W_{1}-C\right)\left(u_{1}^{* \prime}-\right. & \left.i A u_{2}^{*}\right) \\
& -\left[\nu+i A \gamma\left(W_{1}-C\right)\right]\left(u_{1}^{* \prime \prime \prime}-A^{2} u_{1}^{* \prime}-i A u_{2}^{* \prime \prime}+i A^{3} u_{2}^{*}\right) \\
= & \left(\gamma W_{1}^{\prime \prime \prime}-W_{1}^{\prime \prime}\right) u_{2}^{*} .
\end{aligned}
$$

Replacing $u_{1}^{*}$ in Eq. (3.14) by $i u_{2}^{* \prime} / A$ from Eq. (3.13), we obtain

$$
\begin{array}{r}
i A\left(W_{1}-C\right)\left(u_{2}^{* \prime \prime}-A^{2} u_{2}^{*}\right)-\left[\nu+i A \gamma\left(W_{1}-C\right)\right]\left(u_{2}^{* \prime \prime \prime \prime}-2 A^{2} u_{2}^{* \prime \prime}+A^{4} u_{2}^{*}\right) \\
=i A\left(W_{1}^{\prime \prime}-\gamma W_{1}^{\prime \prime \prime}\right) u_{2}^{*} .
\end{array}
$$

Writing Eq. (3.15) in dimensionless form by letting $\eta_{2}=x_{2} / L, V_{1}=W_{1} / W_{0}$, $w_{2}=u_{2}^{*} / W_{0}, \alpha=A L$ and $c=C / W_{0}$ where $L$ and $W_{0}$ are a characteristic length and a characteristic velocity respectively of the steady laminar flow, we arrive at 


$$
\begin{aligned}
i \alpha\left(V_{1}-c\right)\left(w_{2}^{\prime \prime}-\alpha^{2} w_{2}\right)-\left[\frac{1}{R}+\frac{i \alpha}{S}\left(V_{1}-c\right)\right]\left(w_{2}^{\prime \prime \prime \prime}\right. & \left.-2 \alpha^{2} w_{2}^{\prime \prime}+\alpha^{4} w_{2}\right) \\
& =i \alpha\left(V_{1}^{\prime \prime}-\frac{1}{S} V_{1}^{\prime \prime \prime \prime}\right) w_{2},
\end{aligned}
$$

where $R=W_{0} L / \nu$ is the Reynolds' number of the laminar flow, $S=L^{2} / \gamma$ is a nondimensional parameter arising from the presence of non-Newtonian terms in the constitutive equations of the fluid, and primes denote ordinary differentiation with respect to $\eta_{2}$.

We see that if $S \rightarrow \infty$, Eq. (3.16) becomes the familiar Orr-Sommerfeld stability equation.

Finally, in terms of $w_{2}$ the boundary conditions (3.4) and (3.5) become

$$
w_{2}=w_{2}^{\prime}=0 \text { at } \eta_{2}=0 \text { and } \eta_{2}=1 \text {, }
$$

and

$$
w_{2}=w_{2}^{\prime}=0 \text { at } \eta_{2}=0 \text { and } \eta_{2} \rightarrow \infty
$$

respectively.

4. The general theorem. Under the assumption that $S$ is finite and $R$ is infinite, Eq. (3.16) takes the form

$$
\left(V_{1}-c\right)\left(w_{2}^{\prime \prime}-\alpha^{2} w_{2}\right)-\frac{1}{S}\left(V_{1}-c\right)\left(w_{2}^{\prime \prime \prime \prime}-2 \alpha^{2} w_{2}^{\prime \prime}+\alpha^{4} w_{2}\right)=\left(V_{1}^{\prime \prime}-\frac{1}{S} V_{1}^{\prime \prime \prime \prime}\right) w_{2} .
$$

We shall now prove the following theorem.

The existence of a point in the flow field for which $V_{1}^{\prime \prime}-(1 / S) V_{1}^{\prime \prime \prime \prime}$ is equal to zero, is a necessary condition for the amplification of a disturbance.

Regarding $w_{2}$ as a complex variable, we define

$$
M\left(w_{2}\right)=\frac{1}{S} w_{2}^{\prime \prime \prime \prime}-\left(1+\frac{2 \alpha^{2}}{S}\right) w_{2}^{\prime \prime}+\left(\alpha^{2}+\frac{\alpha^{4}}{S}\right) w_{2}+\frac{\left(V_{1}^{\prime \prime}-\frac{1}{S} V_{1}^{\prime \prime \prime \prime}\right)}{V_{1}-c} w_{2}
$$

and

$$
\bar{M}\left(w_{2}\right)=\frac{1}{S} \bar{w}_{2}^{\prime \prime \prime \prime}-\left(1+\frac{2 \alpha^{2}}{S}\right) \bar{w}_{2}^{\prime \prime}+\left(\alpha^{2}+\frac{\alpha^{4}}{S}\right) \bar{w}_{2}+\frac{\left(V_{1}^{\prime \prime}-\frac{1}{S} V_{1}^{\prime \prime \prime \prime}\right)}{V_{1}-\bar{c}} \bar{w}_{2},
$$

where a bar denotes the complex conjugate of the corresponding unbarred quantity. It is easily seen from Eq. (4.1) that both $M\left(w_{2}\right)$ and $\bar{M}\left(w_{2}\right)$ are equal to zero.

To prove the theorem we assume that $V_{1}^{\prime \prime}-(1 / S) V_{1}^{\prime \prime \prime \prime} \neq 0$ throughout the flow field. Then, multiplying $M\left(w_{2}\right)$ by $\bar{w}_{2}$ and $\bar{M}\left(w_{2}\right)$ by $w_{2}$ and subtracting the resulting expressions, we obtain

$$
\begin{aligned}
\bar{w}_{2} M\left(w_{2}\right)-w_{2} \bar{M}\left(w_{2}\right)=\frac{1}{S}\left(\bar{w}_{2} w_{2}^{\prime \prime \prime \prime}\right. & \left.-w_{2} \bar{w}_{2}^{\prime \prime \prime \prime}\right) \\
& -\left(1+\frac{2 \alpha^{2}}{S}\right)\left(\bar{w}_{2} w_{2}^{\prime \prime}-w_{2} \bar{w}_{2}^{\prime \prime}\right) \\
& +\left(V_{1}^{\prime \prime}-\frac{1}{S} V_{1}^{\prime \prime \prime \prime}\right)\left(\frac{1}{V_{1}-c}-\frac{1}{V_{1}-\bar{c}}\right)\left|w_{2}\right|^{2} .
\end{aligned}
$$


Integrating Eq. (4.4) with respect to $\eta_{2}$ between the limits $\eta_{2}=0$ and $\eta_{2}=1$, we have

$$
\begin{gathered}
\int_{0}^{1}\left[\bar{w}_{2} M\left(w_{2}\right)-w_{2} \bar{M}\left(w_{2}\right)\right] d \eta_{2}=\left.\frac{1}{S}\left[\left(\bar{w}_{2} w_{2}^{\prime \prime \prime}-w_{2} \bar{w}_{2}^{\prime \prime \prime}\right)-\left(\bar{w}_{2}^{\prime} w_{2}^{\prime \prime}-w_{2}^{\prime} \bar{w}_{2}^{\prime \prime}\right)\right]\right|_{0} ^{1} \\
-\left.\left(1+\frac{2 \alpha^{2}}{S}\right)\left[\bar{w}_{2} w_{2}^{\prime}-w_{2} \bar{w}_{2}^{\prime}\right]\right|_{0} ^{1}+2 i c_{i} \int_{0}^{1} \frac{\left(V_{1}^{\prime \prime}-\frac{1}{S} V_{1}^{\prime \prime \prime}\right)}{\left|V_{1}-c\right|^{2}}\left|w_{2}\right|^{2} d \eta_{2} .
\end{gathered}
$$

Because of the boundary conditions (3.17), the first two terms on the right hand side of Eq. (4.5) vanish, and further, since both $M\left(w_{2}\right)$ and $\bar{M}\left(w_{2}\right)$ are equal to zero, the left hand side of the equation is equal to zero. It then follows that

$$
c_{i} \int_{0}^{1} \frac{\left(V_{1}^{\prime \prime}-\frac{1}{S} V_{1}^{\prime \prime \prime \prime}\right)}{\left|V_{1}-c\right|^{2}}\left|w_{2}\right|^{2} d \eta_{2}
$$

must vanish. But this is impossible, since $c_{i}>0$, for a disturbance which tends to grow, and hence $\left[1 /\left|V_{1}-c\right|^{2}\right]>0$. Further $\left|w_{2}\right|^{2}$ is positive and by assumption $V_{1}^{\prime \prime}-$ $(1 / S) V_{1}^{\prime \prime \prime \prime} \neq 0$ everywhere in the flow field. Thus we conclude that for a disturbance which tends to grow, there exists an $\eta_{2}, 0<\eta_{2}<1$, for which $V_{1}^{\prime \prime}-(1 / S) V_{1}^{\prime \prime \prime \prime}$ is equal to zero.

In the case of laminar flow by a flat plate the proof of the theorem differ only in that the limits of integration become $\eta_{2}=0$ and $\eta_{2}=\infty$, and the boundary conditions (3.18) are used in place of those given by (3.17).

\section{REFERENCES}

1. Lord Rayleigh, On the stability or instability of certain fluid motions, I, Proc. Lond. Math. Soc. 9, 57-70 (1880); see also Scientific Papers 1, 474-487 (1899)

2. H. Schlichting, Boundary layer theory, translated by J. Kestin, McGraw-Hill, Inc., New York, 1955, pp. 314-323

3. R. S. Rivlin, Further remarks on the stress-deformation relations for isotropic materials, J. Ratl. Mech. and Anal. 4, 681-702 (1955)

4. R. S. Rivlin, Solutions of some problems in the exact theory of visco-elasticity, J. Ratl. Mech. and Anal. 5, 179-188 (1956) 